\title{
Pelvic Congestion Syndrome: A Missed Opportunity
}

\author{
Claire Kaufman ${ }^{1}$ Nancy Ann Little ${ }^{2}$ \\ ${ }^{1}$ Department of Radiology, University of Utah, Salt Lake City, Utah, \\ United States \\ 2 Department of Radiology, University of Wisconsin, Madison, \\ Wisconsin, United States \\ Indian J Radiol Imaging 2021;31:539-544.
}

\begin{abstract}
Address for correspondence Claire Kaufman, MD, Department of Radiology, University of Utah, 30 N 1900 E, Salt Lake City, UT 84132, United States (e-mail: Claire.Kaufman@gmail.com).
\end{abstract}

\begin{abstract}
Background/Aims Chronic pelvic pain (CPP) is pelvic pain for greater than 6 months with many potential causes one being pelvic congestion syndrome (PCS). PCS is diagnosed by clinical symptoms, exclusion of other etiologies, and imaging. Given the complex nature and diagnosis of CPP, we examined ordering and referral patterns in our local population to understand how the imaging findings of PCS correlate with patient symptoms and referral and treatment.

Materials and Methods After IRB approval, we searched all 18 to 70 -year-old females with CT of the pelvis between March 2015 and March 2018 with the terms "pelvic" plus "congestion," "varices," or "vein dilation" in the dictation. Via electronic medical record and image review we collected data regarding demographics, clinical presentation, symptoms, imaging findings, ordering provider, and any follow-up/referrals or interventions regarding PCS.

Results A total of 96 patients were included of the 197 studies, with an average age of 47 years and average body mass index of $19.3(n=93)$ at the time of imaging. The reason for imaging was often acute abdominal or flank pain $(n=22)$ with $38 \%$ of cases ordered from the emergency room. Only 72 patients had documentation of clinical

Keywords

- pelvic venous insufficiency

- pelvic congestion syndrome

- chronic pelvic pain

- embolization evaluation for symptoms of PCS. Notably, only 20 were referred for their symptoms, and only two patients were referred to IR. Both patients underwent successful endovascular intervention.

Conclusion CPP is a common cause of morbidity with PCS representing an underdiagnosed cause. We demonstrate that while imaging findings may be incidental, we are failing to capture and triage patients with clinical symptoms of PCS. Radiologists can play a key role in the care of these patients.
\end{abstract}

\section{Introduction}

Chronic pelvic pain (CPP) is defined as pain in the pelvis for greater than 6 months duration that is not exclusively cyclical or post coital. ${ }^{1,2} \mathrm{CPP}$ can be caused by many different etiologies including endometriosis, fibroids, malignancy, adenomyosis, uterine prolapse, irritable bowel syndrome, dilated/incompetent pelvic veins, or even psychiatric causes. ${ }^{3}$ This often leads to difficulty in diagnosis and subsequent treatment. CPP is a common problem among women of childbearing age with a reported incidence 24 to $39.1 \%^{4-6}$ leading to 10 to $40 \%$ of gynecology visits. ${ }^{1,7}$ Despite the high incidence, studies have shown that a large percentage of women (40-75\%) do not seek medical consultation
DOI https://doi.org/ 10.1055/s-0041-1735497. ISSN 0971-3026.

\footnotetext{
(C) 2021. Indian Radiological Association. All rights reserved. This is an open access article published by Thieme under the terms of the Creative Commons Attribution-NonDerivative-NonCommercial-License, permitting copying and reproduction so long as the original work is given appropriate credit. Contents may not be used for commercial purposes, or adapted, remixed, transformed or built upon. (https://creativecommons.org/ licenses/by-nc-nd/4.0/) Thieme Medical and Scientific Publishers Pvt. Ltd., A-12, 2nd Floor, Sector 2, Noida-201301 UP, India
} 
despite their symptoms. ${ }^{4,8}$ One study showed that $60 \%$ of women who were seen by their general practitioner for CPP did not have any further referral or work-up. ${ }^{9}$ Even for those patients who do seek medical consultation the work-up of CPP can be challenging which may lead to a delay or lack of diagnosis and treatment.

Pelvic venous insufficiency or pelvic congestion syndrome (PCS) is the result of insufficiency of the gonadal and/or pelvic veins. PCS is often seen in multiparous women due to increased blood volume and dilatation of the ovarian and pelvic veins during pregnancy, as well as compression by the gravid uterus. This leads to irreversible dilatation and valve damage causing reflux. ${ }^{10}$ Additionally, $15 \%$ of women lack valves in the ovarian veins which also pre-disposes these vessels to reflux. ${ }^{11}$ PCS has also been associated with compression of central veins in the setting of Nutcracker syndrome, with compression of the left renal vein by the superior mesenteric artery, or May-Thurner, with compression of the left common iliac vein by the right common iliac artery. $^{2,12,13}$

Originally considered a diagnosis of exclusion, the diagnosis of PCS requires appropriate imaging and clinical findings; however, it is not always considered and evaluated during the work-up of CPP. Symptoms of PCS include dull aching pain in the pelvis which can be worse with walking, standing, or prolonged sitting, and at the end of the day. The pain can be worse during menses, intercourse, or postcoital. ${ }^{14}$ Prominent varices on the perineum, buttock, and extremities can be seen. Other associated symptoms are vague and overlap with other etiologies of CPP, further causing a diagnostic dilemma. These include bloating, lethargy, depression, abdominal/pelvic tenderness, rectal discomfort, urinary frequency, vaginal discharge, or even lumbosacral neuropathy. 2,15

Given the challenge in elucidating the underlying cause or causes of CPP, these patients are often not referred for followup and evaluation. The purpose of our longitudinal study was to correlate the clinical symptoms of patients with computed tomography (CT) imaging findings suggestive of PCS. Anticipating that many of these patients may suffer from CPP but are not being referred for further work-up and evaluation of PCS, we also tracked the referral and follow-up patterns.

\section{Materials and Methods}

A retrospective longitudinal review was performed at our tertiary-care hospital. This study was approved by the institutional board review at the institution and performed in compliance with the Health Information Portability and Accountability Act. All female patients aged 18 to 70 years who underwent a CT or computed tomography angiography of the abdomen and pelvis from March 2015 to March 2018 for which the final CT dictation included the terms "pelvic" plus "congestion," "varices," or "vein dilation" were pulled from the picture archiving and communication system (PACS). Exclusion criteria included duplicate exams, patients who had already undergone intervention for PCS, findings of venous congestion elsewhere (hepatic, rectal, mesenteric stranding, etc.), imaging findings that were not consistent with PCS or gonadal/pelvic vein dilation despite mention in the dictation, or studies with notation of findings negative for PCS (-Fig. 1).

The electronic medical record (EMR) was reviewed, and data was collected regarding patient demographics, clinical presentation, prior symptoms, imaging findings, ordering study provider, and any follow-up referral or interventions for CPP or PCS. Patient age, body mass index (BMI), parity and menstruation status were obtained. Symptoms associated with PCS including CPP, pelvic pressure, dyspareunia, acute pelvic pain ( $<6$ months), back pain, menorrhagia, lower extremity varices, and vulvar varices were documented. Additionally, imaging findings including location of dilated veins, findings suggestive of nutcracker, or other gynecologic pathology were documented. The ordering department and reason for the exam were noted. Finally, it was documented whether pelvic pain or the potential diagnosis of PCS was addressed in follow-up via attendance at a subsequent appointment or referral to a specialist (ob/gyn, interventional radiology, or vascular surgery). Findings of this longitudinal study are descriptive and therefore no statistical analysis was applied.

\section{Results}

There were 197 studies which met the search criteria. Subsequently 101 were excluded given the exclusion criteria described above. The included patient population demographics $(n=96)$ are described in - Table 1 , with an average age of 47 years and average BMI of $19.3 \mathrm{~kg} / \mathrm{m}^{2}$. The majority were premenopausal (43 of 67 patients with documented menopausal status) and parous (50 of 57 patients with documented parity). Only 68 patients had documentation of the presence or absence of symptoms classically associated with PCS in the EMR; 54 of these patients endorsed one or more symptom. Pelvic pain ( $<6$-month duration) and back pain were the most common symptom documented in the EMR. The indication for imaging studies is shown in -Table 2; most studies were obtained for abdominal pain. "Other" reasons for the imaging includes trauma, DVT, and aortic disease.

The majority of patients had dilated pelvic veins bilaterally $(n=58)$ while 35 patients had isolated left gonadal or pelvic varices. Only three patients had isolated right pelvic varices; one had varices seen originating from the right common femoral extending to the right labia without dilatation of the ovarian vein, one had isolated varices in the right broad ligament, and the last had portal hypertension, chronic portal vein occlusion, and large portosystemic collaterals involving the right ovarian vein but not the left.

The ordering department of the included imaging studies, as well as subsequent referrals is described in - Table 3 . Emergency and primary care providers accounted for approximately half of imaging orders (38 and 17\%, respectively), but a variety of other providers, ranging from oncologists to gastroenterologists, also ordered studies. Only 20 of 96 patients with imaging suggestive of PCS were referred to one 

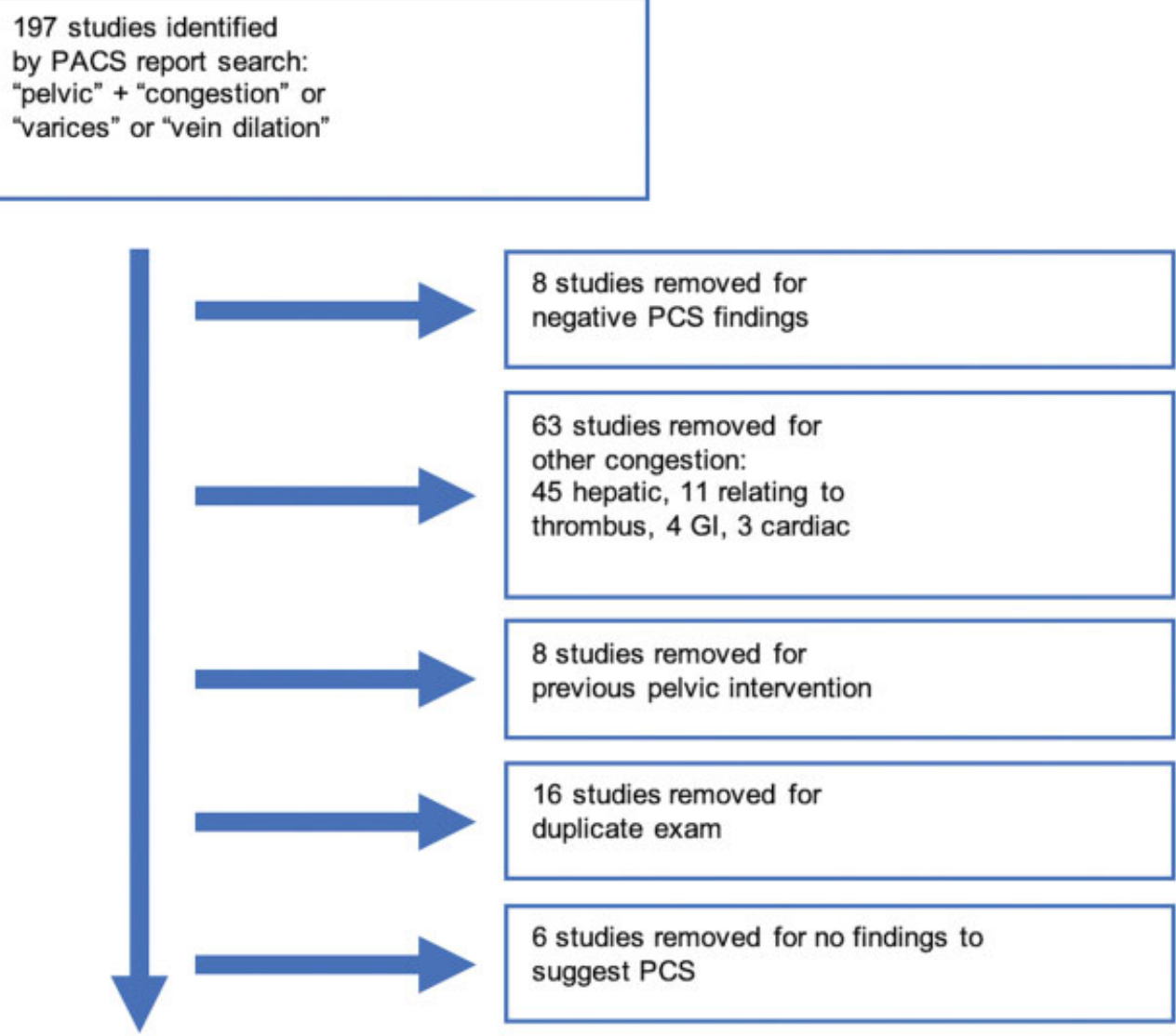

96 studies representing

96 unique patients with imaging evidence of PCS

Fig. 1 Detailed flowchart of included and excluded patients.

or more providers for follow-up to address these imaging findings. The vast majority (85\%) of these patients were referred to ob/gyn. Only two patients were referred to interventional radiology ( - Fig. 2), while another four were referred to vascular surgery.

\section{Discussion}

CPP is a common problem in menstruating women that leads to a large number of gynecology referrals. It is difficult to diagnose and treat due to the many differing etiologies. CPP not only causes physical pain and discomfort but also can cause great psychological stress due to the negative connotations and stereotypes associated with pelvic pain. CPP due to ovarian varicosities was first reported in $1958 .{ }^{16} \mathrm{PCS}$ is CPP characterized by pressure, aching, or heaviness in the pelvis that worsens with standing, dyspareunia, and dysmenorrhea.

The work-up for PCS is difficult as the imaging findings alone are not diagnostic but suggestive or confirmatory in the appropriate clinical setting. While venography is considered the gold-standard, often CT, MRI, or ultrasound are used to suggest a diagnosis of PCS with findings of dilated gonadal and parametrial vessels. On CT the pelvic varicosities will appear as dilated, tubular, enhancing parametrial structures. One proposed diagnostic criteria on $\mathrm{CT}$ is at least four ipsilateral pelvic varicosities measuring $>4 \mathrm{~mm}$ diameter or dilation of the ovarian vein of $>8 \mathrm{~mm}$ diameter, ${ }^{17}$ however, this is rarely used in practice and findings are often described subjectively. ${ }^{15}$

Conservative treatment of PCS with medication can be attempted; gonadotropin-releasing hormone analogs or medroxyprogesterone acetate have been utilized with the intent to reduce hormonal changes and subsequent fluctuation of vascular flow; however, relief is usually temporary. ${ }^{2}$ Surgical treatment for PCS includes hysterectomy and laparoscopic ligation of the ovarian veins. Although logically resection of the uterus should remove the source of pain, studies have shown that $33 \%$ of women report residual pain and $20 \%$ of women have recurrent pain after hysterectomy. ${ }^{18,19}$ Literature supporting ligation of the ovarian vein is limited to case reports and small case series. ${ }^{20-23}$

While there is a lack of randomized controlled trials and high-quality data, there is a consistent evidence published 
Table 1 Patient characteristics

\begin{tabular}{|c|c|}
\hline Age @ imaging (years) & 46.9 \\
\hline BMI @ imaging $\left(\mathrm{kg} / \mathrm{m}^{2}\right)$ & 19.3 \\
\hline \multicolumn{2}{|l|}{ Menopause $(n=67)$} \\
\hline Pre & $64 \%(n=43)$ \\
\hline Post & $36 \%(n=24)$ \\
\hline \multicolumn{2}{|l|}{ Gravidity $(n=57)$} \\
\hline G0 & $12 \%(n=7)$ \\
\hline G1+ & $88 \%(n=50)$ \\
\hline \multicolumn{2}{|l|}{ Delivery $(n=49)$} \\
\hline Vaginal & $8 \%(n=4)$ \\
\hline Cesarean & $25 \%(n=12)$ \\
\hline Not reported & $67 \%(n=33)$ \\
\hline \multicolumn{2}{|l|}{ Clinical symptoms $(n=54)$} \\
\hline Chronic pelvic pain & $n=9$ \\
\hline Pelvic pressure & $n=3$ \\
\hline Dyspareunia & $n=8$ \\
\hline Pelvic pain (<6 mo) & $n=22$ \\
\hline Back pain & $n=33$ \\
\hline Menorrhagia & $n=17$ \\
\hline Lower extremity varies & $n=3$ \\
\hline Vulvar varices & $n=3$ \\
\hline \multicolumn{2}{|l|}{ Imaging findings $(n=96)$} \\
\hline Right dilated pelvic veins & $n=3$ \\
\hline Left dilated pelvic veins & $n=35$ \\
\hline Bilateral dilated pelvic veins & $n=58$ \\
\hline Nutcracker & $n=8$ \\
\hline Other ob/gyn pathology & $n-11$ \\
\hline
\end{tabular}

Table 2 Indication for CT study

\begin{tabular}{|l|l|}
\hline Indication & $\mathbf{n = 9 6}$ \\
\hline Abdominal pain, lower & $26 \%$ \\
\hline Abdominal pain, upper & $9 \%$ \\
\hline Abdominal pain, nonfocal & $16 \%$ \\
\hline Cancer (surveillance, staging) & $27 \%$ \\
\hline GU symptoms & $4 \%$ \\
\hline Pelvic/vulvar varicosities & $2 \%$ \\
\hline Oher & $16 \%$ \\
\hline
\end{tabular}

Table 3 Ordering department and subsequent patient referral for PCS

\begin{tabular}{|l|l|l|}
\hline & Ordering $(\boldsymbol{n}=\mathbf{9 6})$ & Referral $(\boldsymbol{n}=\mathbf{2 0})$ \\
\hline $\begin{array}{l}\text { Emergency } \\
\text { Department }\end{array}$ & $38 \%$ & - \\
\hline Primary care & $17 \%$ & $10 \%$ \\
\hline Ob/Gyn & $3 \%$ & $85 \%$ \\
\hline Radiology & $2 \%$ & $10 \%$ \\
\hline Vascular surgery & - & $20 \%$ \\
\hline Other & $41 \%$ & $15 \%$ \\
\hline
\end{tabular}

across multiple different specialties suggesting that embolization of dilated or refluxing gonadal and pelvic veins is efficacious at relieving pain symptoms in PCS patients with low rates of complications and high technical success. Two recent literature reviews published in 2016, one looking at 20 studies with 1,081 patients ${ }^{24}$ and another with 20 case series including 1,308 patients, ${ }^{25}$ showed significant relief in patient symptoms with low rates of complications. Recurrence rates are reported at $8 \% .{ }^{15}$ Gynecology literature also supports embolization for PCS for example, one study reports
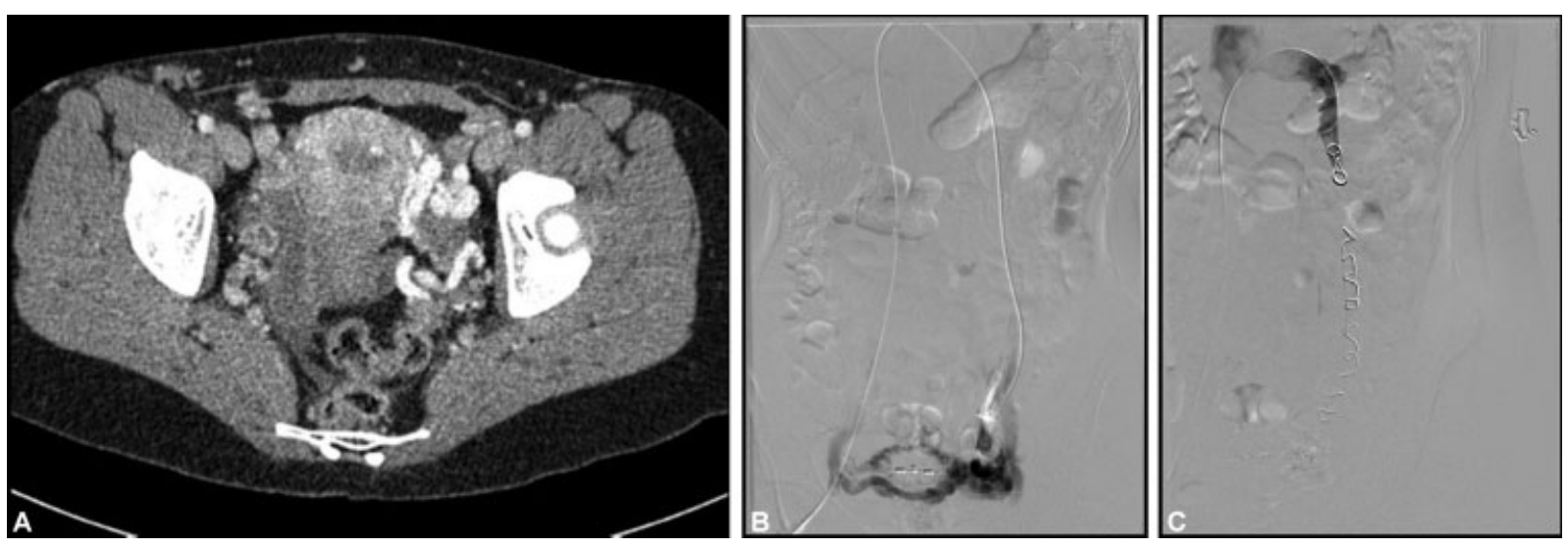

Fig. 2 Images obtained from a premenopausal multiparous female initially evaluated for pelvic pain (pressure sensation improved with standing, dyspareunia). Patient was referred to our group by an outside Ob/Gyn provider. (A) CT abd/pelvis w IV contrast shows multiple dilated and tortuous parametrial veins, present bilaterally but more significant on the left. (B) Venogram shows prominent pelvic collateral vessels and reflux. (C) Post coiling venogram demonstrates occlusion of the ovarian vein without reflux. 
technical and clinical success rate of $100 \%$ as defined by the visual analog scale. ${ }^{26}$ The most recent clinical practice guideline from the American Venous Forum also supports ovarian vein embolization as a treatment for PCS. ${ }^{27}$

We found that only $71 \%$ (68 of 96) of patients with imaging evidence of PCS had documentation of evaluation of symptoms that can be seen in the setting of PCS. Of those patients, $79 \%$ ( 54 of 68 ) had positive findings of one or more symptoms of PCS. This indicates two things; first, these patients are often not being evaluated for symptoms that can be caused by PCS. This may be related to the reason for the CT scan. For example, an oncology follow-up study may not necessarily trigger a clinician to ask about symptoms of CPP. However, these findings are in line with literature showing that women do not always seek care for CPP. A U.S. population-based study showed that $75 \%$ of women with bothersome CPP had not seen a health care provider for these symptoms. ${ }^{8}$ The second factor is that imaging findings alone are not sufficient to diagnose PCS. Anatomical studies have shown that valves are absent in $15 \%$ of left and $6 \%$ of right ovarian veins with incompetent valves in $41 \%$ of left and $46 \%$ of right ovarian veins. ${ }^{28}$ As venous capacity of the pelvic veins can increase over 60 -fold during pregnancy, ovarian and pelvic varicosities are frequently seen in multiparous women. ${ }^{29,30}$ Women may have incidentally noted pelvic varicosities without clinical symptoms consistent with PCS. In these cases, no further work-up would be indicated.

However, of the 54 patients with imaging findings and history suggestive of PCS only 20 patients were subsequently referred for further work-up. The most common referral was to ob/gyn $(n=17)$. This is appropriate as there are many etiologies for CPP and a complete multi-disciplinary work-up should be obtained even when PCS is suspected. However, only two patients out of the 54 were referred to interventional radiology. Both of these women subsequently underwent successful embolization. None of the patients referred to vascular surgery $(n=4)$ underwent intervention with the surgeons. This lack of referral demonstrates the continued challenge posed by lack of knowledge and awareness of PCS and potential treatment options. This highlights a need for outreach by interventional radiologists to referring providers who are ordering these imaging studies, as well as to patients.

\section{Conclusion}

CPP is a common cause of morbidity in women. Our longitudinal study demonstrated that while imaging findings are seen in women even without documented symptoms we are failing to capture and triage a large number of patients with both imaging and clinical symptoms of PCS. Radiologists can play a key role in the care and triage of these patients. Via increased awareness and outreach, we have the opportunity to intervene and relieve the symptoms of many patients.

Note

This study was presented at the Society of Interventional Radiology 2019 Annual Meeting as a poster.
Funding

None.

Conflict of Interest

None declared.

\section{References}

1 Robinson JC. Chronic pelvic pain. Curr Opin Obstet Gynecol 1993; 5(06):740-743

2 Ignacio EA, Dua R, Sarin S, et al. Pelvic congestion syndrome: diagnosis and treatment. Semin Intervent Radiol 2008;25(04): 361-368

3 Laborda A, Medrano J, de Blas I, Urtiaga I, Carnevale FC, de Gregorio MA. Endovascular treatment of pelvic congestion syndrome: visual analog scale (VAS) long-term follow-up clinical evaluation in 202 patients. Cardiovasc Intervent Radiol 2013;36 (04):1006-1014

4 Zondervan KT, Yudkin PL, Vessey MP, et al. The community prevalence of chronic pelvic pain in women and associated illness behaviour. Br J Gen Pract 2001;51(468):541-547

5 Venbrux AC, Chang AH, Kim HS, et al. Pelvic congestion syndrome (pelvic venous incompetence): impact of ovarian and internal iliac vein embolotherapy on menstrual cycle and chronic pelvic pain. J Vasc Interv Radiol 2002;13(2 Pt 1):171-178

6 Jamieson DJ, Steege JF. The prevalence of dysmenorrhea, dyspareunia, pelvic pain, and irritable bowel syndrome in primary care practices. Obstet Gynecol 1996;87(01):55-58

7 El-Sadr AR, Mina E. Anatomical and surgical aspects in the operative management of varicocele. Urol Cutaneous Rev 1950; 54(05):257-262

8 Mathias SD, Kuppermann M, Liberman RF, Lipschutz RC, Steege JF. Chronic pelvic pain: prevalence, health-related quality of life, and economic correlates. Obstet Gynecol 1996;87(03):321-327

9 Zondervan KT, Yudkin PL, Vessey MP, Dawes MG, Barlow DH, Kennedy SH. Patterns of diagnosis and referral in women consulting for chronic pelvic pain in UK primary care. Br J Obstet Gynaecol 1999;106(11):1156-1161

$10 \mathrm{Koo}$ S, Fan CM. Pelvic congestion syndrome and pelvic varicosities. Tech Vasc Interv Radiol 2014;17(02):90-95

11 Freedman J, Ganeshan A, Crowe PM. Pelvic congestion syndrome: the role of interventional radiology in the treatment of chronic pelvic pain. Postgrad Med J 2010;86(1022):704-710

12 Liddle AD, Davies AH. Pelvic congestion syndrome: chronic pelvic pain caused by ovarian and internal iliac varices. Phlebology 2007;22(03):100-104

13 Scultetus AH, Villavicencio JL, Gillespie DL. The nutcracker syndrome: its role in the pelvic venous disorders. J Vasc Surg 2001;34 (05):812-819

14 Kim HS, Malhotra AD, Rowe PC, Lee JM, Venbrux AC. Embolotherapy for pelvic congestion syndrome: long-term results. J Vasc Interv Radiol 2006;17(2 Pt 1):289-297

15 Phillips D, Deipolyi AR, Hesketh RL, Midia M, Oklu R. Pelvic congestion syndrome: etiology of pain, diagnosis, and clinical management. J Vasc Interv Radiol 2014;25(05):725-733

16 Topolanski-Sierra R. Pelvic phlebography. Am J Obstet Gynecol 1958;76(01):44-52

17 Coakley FV, Varghese SL, Hricak H. CT and MRI of pelvic varices in women. J Comput Assist Tomogr 1999;23(03):429-434

18 Beard RW, Kennedy RG, Gangar KF, et al. Bilateral oophorectomy and hysterectomy in the treatment of intractable pelvic pain associated with pelvic congestion. Br J Obstet Gynaecol 1991;98 (10):988-992

19 Carter JE. Surgical treatment for chronic pelvic pain. JSLS 1998;2 (02):129-139

20 Gargiulo T, Mais V, Brokaj L, Cossu E, Melis GB. Bilateral laparoscopic transperitoneal ligation of ovarian veins for treatment of 
pelvic congestion syndrome. J Am Assoc Gynecol Laparosc 2003; 10(04):501-504

21 Rogers A, Beech A, Braithwaite B. Transperitoneal laparoscopic left gonadal vein ligation can be the right treatment option for pelvic congestion symptoms secondary to nutcracker syndrome. Vascular 2007;15(04):238-240

22 Stones RW. Pelvic vascular congestion-half a century later. Clin Obstet Gynecol 2003;46(04):831-836

23 Takeuchi K, Mochizuki M, Kitagaki S. Laparoscopic varicocele ligation for pelvic congestion syndrome. Int J Gynaecol Obstet 1996;55(02):177-178

24 Mahmoud O, Vikatmaa P, Aho P, et al. Efficacy of endovascular treatment for pelvic congestion syndrome. J Vasc Surg Venous Lymphat Disord 2016;4(03):355-370

25 Daniels JP, Champaneria R, Shah L, Gupta JK, Birch J, Moss JG. Effectiveness of embolization or sclerotherapy of pelvic veins for reducing chronic pelvic pain: a systematic review. J Vasc Interv Radiol 2016;27(10):1478-1486.e8
26 Nasser F, Cavalcante RN, Affonso BB, Messina ML, Carnevale FC, de Gregorio MA. Safety, efficacy, and prognostic factors in endovascular treatment of pelvic congestion syndrome. Int J Gynaecol Obstet 2014;125(01):65-68

27 Gloviczki P, Comerota AJ, Dalsing MC, et al; Society for Vascular Surgery American Venous Forum. The care of patients with varicose veins and associated chronic venous diseases: clinical practice guidelines of the Society for Vascular Surgery and the American Venous Forum. J Vasc Surg 2011; 53(Suppl 5):2S-48S

28 Ahlberg NE, Bartley O, Chidekel N. Right and left gonadal veins. An anatomical and statistical study. Acta Radiol Diagn (Stockh) 1966; 4(06):593-601

29 Hodgkinson CP. Physiology of the ovarian veins during pregnancy. Obstet Gynecol 1953;1(01):26-37

30 Durham JD, Machan L. Pelvic congestion syndrome. Semin Intervent Radiol 2013;30(04):372-380 